\title{
\begin{tabular}{l|l} 
Mibraries & DSpace@MIT
\end{tabular}
}

\author{
MIT Open Access Articles
}

\section{Slippery Bottom Boundary Layers: The Loss of Energy From the General Circulation by Bottom Drag}

The MIT Faculty has made this article openly available. Please share how this access benefits you. Your story matters.

Citation: Ruan, Xiaozhou, Wenegrat, Jacob 0. and Gula, Jonathan. 2021. "Slippery Bottom Boundary Layers: The Loss of Energy From the General Circulation by Bottom Drag." Geophysical Research Letters, 48 (19).

As Published: http://dx.doi.org/10.1029/2021gl094434

Publisher: American Geophysical Union (AGU)

Persistent URL: https://hdl.handle.net/1721.1/140534

Version: Author's final manuscript: final author's manuscript post peer review, without publisher's formatting or copy editing

Terms of Use: Article is made available in accordance with the publisher's policy and may be subject to US copyright law. Please refer to the publisher's site for terms of use. 


\title{
Slippery bottom boundary layers: the loss of energy from the general circulation by bottom drag
}

\author{
Xiaozhou Ruan ${ }^{1}$, Jacob O. Wenegrat ${ }^{2}$, Jonathan Gula ${ }^{3,4}$ \\ ${ }^{1}$ Department of Earth, Atmospheric, and Planetary Sciences, Massachusetts Institute of Technology, \\ Cambridge, Massachusetts \\ ${ }^{2}$ Department of Atmospheric and Oceanic Science, University of Maryland, College Park, Maryland \\ ${ }^{3}$ Université de Brest, CNRS, IRD, Ifremer, Laboratoire d'Océanographie Physique et Spatiale (LOPS), \\ IUEM, Brest,France \\ ${ }^{4}$ Institut Universitaire de France (IUF)
}

\section{Key Points:}

- Thermal wind shear in the bottom boundary layer reduces the magnitude of the near-bottom flow and bottom stress

- Accounting for boundary layer shear reduces kinetic energy loss from the geostrophicallybalanced flow by more than $50 \%$

- Observations and models that do not resolve the bottom boundary layer will overestimate the energy dissipation due to bottom drag

Corresponding author: Xiaozhou Ruan, xruan@mit.edu

This is the author manuscript accepted for publication and has undergone full peer review but has not been through the copyediting, typesetting, pagination and proofreading process, which may lead to differences between this version and the Version of Record. Please cite this article as doi: 10.1029/2021GL094434.

This article is protected by copyright. All rights reserved. 


\begin{abstract}
Bottom drag is believed to be one of the key mechanisms that remove kinetic energy from the ocean's general circulation. However, large uncertainty still remains in global estimates of bottom drag dissipation. One significant source of uncertainty comes from the velocity structures near the bottom where the combination of sloping topography and stratification can reduce the mean flow magnitude, and thus the bottom drag dissipation. Using high-resolution numerical simulations, we demonstrate that previous estimates of bottom drag dissipation are biased high because they neglect velocity shear in the bottom boundary layer. The estimated bottom drag dissipation associated with geostrophic flows over the continental slopes is at least $56 \%$ smaller compared with prior estimates made using total velocities outside of the near-bottom layer. The diagnostics suggest the necessity of resolving the bottom boundary layer structures in coarse-resolution ocean models and observations in order to close the global kinetic energy budget.
\end{abstract}

\title{
Plain Language Summary
}

When an oceanic flow is close to the seafloor, the bottom drag converts its kinetic energy (KE) to heat loss through viscous friction, and this dissipation of KE has been shown to be very sensitive to the magnitude of the flow. Despite previous estimates indicating the bottom drag being a significant mechanism for removing KE from the ocean's general circulation, large uncertainty still remains. Using high-resolution numerical simulations of the Atlantic Ocean, we demonstrate that accounting for the velocity reduction through the oceanic bottom boundary layer reduces kinetic energy loss from the balanced flow (in which the pressure gradient force and Coriolis force balance) by at least $56 \%$ over the continental slopes. This velocity reduction is due to the presence of sloping topography and ocean stratification near the bottom, which should be resolved in future observational and modeling efforts toward a more complete picture of the ocean's energy budget.

\section{Introduction}

The ocean circulation is continually energized by external forcing, with an estimated $\sim 0.8-1 \mathrm{TW}$ of mechanical energy input into the general circulation by wind work on the geostrophic flow (Ferrari \& Wunsch, 2009). This kinetic energy (KE) input must be balanced by dissipation at molecular scales for the ocean energy budget to remain in equilibrium; however, identifying the mechanisms through which this occurs remains an ongoing challenge in oceanography. Several of the most prominent candidates for removing KE from the ocean circulation involve flow over bottom topography, where energy can be removed from the balanced-flow directly through the quadratic bottom boundary layer drag (Sen et al., 2008; Arbic et al., 2009; Scott \& Xu, 2009; C. J. Wright et al., 2013), by the generation and breaking of internal waves over rough topography (Nikurashin \& Ferrari, 2011; Scott et al., 2011; Trossman et al., 2013; C. J. Wright et al., 2014; Trossman et al., 2016), or through the formation of submesoscale instabilities (Gula et al., 2016; Ruan et al., 2017; Wenegrat et al., 2018; Wenegrat \& Thomas, 2020). In this manuscript we focus on the first of these mechanisms, energy loss to quadratic bottom boundary layer drag, and specifically how prior estimates of this may be significantly biased by unresolved bottom boundary layer (BBL) processes. For simplicity, we will refer to quadratic bottom boundary layer drag as bottom drag hereafter.

Constraining the global contribution of bottom drag to the ocean energy budget is challenging due to the range of space and timescales involved. Sen et al. (2008) used a set of 290 deep-water moorings in conjunction with satellite altimetry to estimate a global (omitting seas shallower than $3000 \mathrm{~m}$ ) sink of KE due to bottom drag of $0.2-$ $0.8 \mathrm{TW}$, with the range representing uncertainty due to spatial biases in the mooring locations and the vertical structure of deep-currents. This range is consistent with a more 
recent estimate of $0.3 \mathrm{TW}$ from more extensive mooring and satellite observations (Huang $\& \mathrm{Xu}, 2018$ ), and with approaches combining moored observations with numerical models that suggest a global integral of $0.1-0.7$ TW (Arbic et al., 2009; C. J. Wright et al., 2013). Despite the significant remaining quantitative uncertainty in the global integrals, these estimates all point to bottom drag as playing a key role in the energetics of ocean circulation. Beyond the integrated budget, bottom drag also exerts a strong control on the energetics of mesoscale eddies - which account for about $90 \%$ of the total ocean KE (Ferrari \& Wunsch, 2009) — with simulations suggesting effects of bottom drag on eddy size, baroclinicity, and the flux of energy between spatial scales (Thompson \& Young, 2006; Arbic \& Flierl, 2004; Arbic \& Scott, 2008; Trossman et al., 2017).

An important limitation in many observational and modeling investigations of bottom drag is that they do not fully resolve the vertical structure of the BBL, where the magnitude of the velocity can decrease rapidly towards the bottom through a combination of thermal wind shear and ageostrophic/frictional flows. Conceptually, this neglect may be argued as justifiable under the assumption of a steady-state energy budget for the BBL, in which case input of energy to the BBL by drag on the interior flow is by definition balanced by dissipative terms. However, when the bottom is not flat, the alongisobath component of the bottom stress generates cross-isobath Ekman transport of buoyancy, which alters this conceptual picture in several important ways as detailed below. Most critically, vertical shear in the BBL acts to reduce the strength of the bottom velocities and drag, generating what MacCready and Rhines (1993) termed 'slippery' BBLs through the process of Ekman buoyancy arrest. The cubic dependence of bottom drag dissipation on bottom velocities suggests that even modest reductions in bottom velocities will have large impact on the bottom drag dissipation, an additional source of quantitative uncertainty not captured in the range of previously published estimates.

In this manuscript we therefore use a high-resolution numerical model of the Atlantic to quantify the extent to which shear in the BBL reduces KE dissipation due to bottom drag. The manuscript is organized as follows. In section 2 we define the bottom drag and measures of dissipation we use to quantify changes due to BBL processes. We then compute these quantities using a high-resolution simulation of the Atlantic: we first focus on the western North Atlantic to illuminate physical processes in section 3, and then the findings are extended to the full Atlantic in section 4. Finally, in section 5 we interpret the results in light of previous global estimates of the KE dissipation due to bottom drag, arguing that these prior estimates are likely biased high by at least a factor of two. These findings provide guidance towards future observational assessments of the role of bottom drag in the ocean general circulation, and we outline a potential strategy for parameterizing these effects in ocean general circulation models.

\section{Bottom drag dissipation and Ekman buoyancy arrest}

The bottom stress, or bottom drag, is defined using the velocity shear at the seafloor:

$$
\left.\boldsymbol{\tau}_{\boldsymbol{b}} \equiv \rho_{0} \nu \frac{\partial \boldsymbol{u}}{\partial z}\right|_{z=0},
$$

where $\nu$ is the molecular viscosity, $\rho_{0}$ is a reference density, and $\boldsymbol{u}(z)$ is the velocity vector parallel to the seafloor. Due to the practical difficulties in measuring velocity shear at the bottom, $\tau_{b}$ is typically calculated using an empirical quadratic drag law

$$
\tau_{\boldsymbol{b}}=\rho_{0} C_{d}\left|\boldsymbol{V}_{\boldsymbol{b}}\right| \boldsymbol{V}_{\boldsymbol{b}},
$$

where $C_{d}$ is a drag coefficient and $\boldsymbol{V}_{\boldsymbol{b}}$ is the mean flow vector close to the bottom. At this point, the correct bottom stress can be reproduced with a bottom velocity defined at any specified depth as long as $C_{d}$ is defined appropriately. In some numerical models (also the case here), $C_{d}$ is parameterized with a logarithmic law of the wall assuming that the lowest grid point is within the logarithmic layer. 
Taylor (1920) further proposed to estimate the KE dissipation within the BBL, $\mathcal{D}$, as the dot product of the bottom stress and bottom velocity $\boldsymbol{V}_{\boldsymbol{b}}$, the famous cubic relationship as used in previous global estimates:

$$
\mathcal{D} \approx \tau_{\boldsymbol{b}} \cdot V_{\boldsymbol{b}}=\rho_{0} C_{d} V_{b}^{3}
$$

where we denote the velocity magnitude as $V_{b}=\left|\boldsymbol{V}_{\boldsymbol{b}}\right|$. In the application of this bulk dissipation formula, $V_{b}$ can no longer be chosen at any arbitrary level but instead has to be taken very close to the seafloor, a distance typically much thinner than the BBL thickness. It has been shown that Taylor's formula provides a reasonable approximation of the true dissipation rate with an appropriate $V_{b}$ when the seafloor is smooth (Ruan, 2021). Note that in some numerical models with a parameterized bottom stress instead of a no-slip bottom boundary condition, the two sides of the equation above balance exactly. For the remainder of this paper, we will call $\mathcal{D}$ the bottom drag dissipation.

Previous global estimates of the bottom drag dissipation of the eddying general circulation can suffer from two main sources of error. First, although low-pass filters are generally applied to the velocity measurements, there is not always a clear distinction between the total low-passed flow and its geostrophic component (e.g. 72-hour used by Sen et al. (2008) and Arbic et al. (2009)). This distinction becomes important when comparing with the KE input rate at the sea surface where the geostrophically-balanced flows are calculated from altimetry. In this manuscript we will therefore also specifically consider the drag on the geostrophic component of the flow,

$$
\mathcal{D}_{g} \approx \tau_{\boldsymbol{b}} \cdot \boldsymbol{V}_{\boldsymbol{g} \boldsymbol{b}}=\rho_{0} C_{d} V_{b}^{2} V_{g b},
$$

where $\boldsymbol{V}_{\boldsymbol{g} \boldsymbol{b}}$ is the geostrophic flow diagnosed directly from the pressure gradient field. Estimates of $D_{g}$ can be compared to $D$ as a measure of ageostrophic effects in the BBL.

The other source of potential error in prior estimates of bottom drag dissipation is the assumption that $D$ can be estimated using measurements several tens to hundreds of meters away from the bottom. This assumption - which is also implicit in many numerical models with unresolved BBLs - can be framed conceptually as an assumption of steady-state BBL energetics, such that energy lost to the interior flow through bottom drag is balanced through dissipative terms (cf. Umlauf et al., 2015). This assumption is likely approximately valid over flat topography with horizontal density surfaces, in which case only the frictional forces within the bottom Ekman layer can modify the near-bottom mean flow magnitude. However, when the bottom is not flat the along-isobath component of the bottom stress generates cross-isobath Ekman transport of buoyancy, which alters this conceptual picture in two important ways. First, the Ekman buoyancy transport leads to an increase of available potential energy (APE) in the BBL, such that $10 \%-50 \%$ of the energy lost from the interior flow by bottom drag goes to APE in the BBL rather than to irreversible dissipation (Umlauf et al., 2015). Secondly, at subinertial timescales the buoyancy anomalies generated by the cross-isobath Ekman transport adjust to thermal wind balance, thereby directly reducing the strength of the bottom velocities and drag, generating what MacCready and Rhines (1993) termed 'slippery' BBLs through the process of Ekman buoyancy arrest. Observational estimates using velocities well above the bottom, and numerical models with coarse vertical resolution near the bottom, will not capture these effects.

Importantly, we emphasize that our focus here is not on the steady state of the Ekman arrest adjustment-which 1D theories suggest can have characteristic timescales of days to years depending on slope angle, stratification, and Coriolis frequency (MacCready \& Rhines, 1991; Brink \& Lentz, 2010)—but rather a more physically relevant "partial" arrest state where there is some reduction of the mean flow magnitude through the BBL. The cubic dependence of the bottom drag on bottom velocity (equation 3) suggests that even small reductions of bottom velocity may have significant impacts on the energetics. This finding has been confirmed recently by Ruan et al. (2019) who used large-eddy 
simulations to show that the bottom friction velocity scaled linearly with the change in geostrophic velocity over the boundary layer depth - indicating that even in cases where the BBL is far from full Ekman arrest the reduction in bottom velocity due to thermal wind shear may significantly reduce KE dissipation within the BBL. In the following sections we therefore use realistic high-resolution simulations of the Atlantic ocean to quantify the effect of BBL shear on basin-scale estimates of bottom drag dissipation.

\section{Effect of boundary layer processes on bottom drag dissipation}

We use a high resolution numerical model of the Atlantic (Fig. 1) to illustrate the effect of BBL processes on the velocity structures and dissipation estimate. The Atlantic wide simulation GIGATL3 is performed with the Coastal and Regional Ocean COmmunity model (CROCO), which is built upon the Regional Oceanic Modeling System (ROMS, Shchepetkin \& McWilliams, 2005). It solves the free surface, hydrostatic, and primitive equations using terrain-following vertical coordinates. The simulation domain covers the full Atlantic Ocean with a horizontal resolution that varies between 3 and $3.5 \mathrm{~km}$ and with 100 vertical levels. The simulation is run from January 2004 to December 2014. Initial and boundary conditions are supplied by the Simple Ocean Data Assimilation (SODA, Carton \& Giese, 2008). The simulation is forced by hourly atmospheric forcings from the Climate Forecast System Reanalysis (CFSR, Saha et al., 2010). Tides are not included in this model solution, which could affect the quantitative estimates of bottom drag dissipation in shallow seas by direct interaction with geostrophic flows (e.g. Rocha et al., 2018). The bathymetry is taken from the SRTM30plus dataset (Becker et al., 2009). The $k-\epsilon$ turbulence closure scheme is used to parameterize vertical mixing, and the Canuto A stability function formulation is applied (Canuto et al., 2001; Umlauf \& Burchard, 2003). There is no explicit lateral diffusivity in the simulation. The effect of bottom friction is parameterized through a logarithmic law of the wall with a roughness length $Z_{0}=0.01$ m.

For computational convenience, we start by examining the model output (i.e. velocities and BBL thickness) in a sub-domain covering the continental slope in the north Atlantic where the deep western boundary current flows along the continental margin (Fig. 1). The BBL thickness is defined using a density threshold such that the top of the BBL collocates with a density decrease of $0.01 \mathrm{~kg} \mathrm{~m}^{-3}$ from the bottom-most grid point, although other definitions are tested as discussed below. Based on this definition, the BBL thickness is generally tens of meters to $O(100) \mathrm{m}$ over the continental shelf with the maximum BBL thickness exceeding $300 \mathrm{~m}$ over the steep slopes (Fig. 1c). These values are much larger than the Ekman depth, which typically does not exceed $O(10) \mathrm{m}$. The interior current speed over the study region is $O(0.1) \mathrm{m} / \mathrm{s}$ (Fig. 1a, b).

We are primarily concerned with the difference between the bottom drag dissipation estimates with and without a resolved BBL. We define two ratios

$$
\begin{gathered}
\mathcal{R}=\frac{\int V_{b}^{2} \cdot V_{g b} d A}{\int V_{\infty}^{3} d A}, \\
\mathcal{R}_{g}=\frac{\int V_{g b}^{3} d A}{\int V_{\infty}^{3} d A},
\end{gathered}
$$

to demonstrate this discrepancy. Here $A$ is area, $V_{\infty}$ is the total velocity linearly interpolated on top of the BBL and $V_{b}$ and $V_{\mathrm{gb}}$ are the total and geostrophic velocities at the bottom-most grid point in the model, which is typically 10-20 m (depending on the thickness of the bottom-most grid cell) away from the bottom topography over the study region (Fig. S1). Similar to previous estimates using mooring records, the diagnostics here are obtained from 5-day averaged velocities to remove high-frequency signals.

$\mathcal{R}$ and $\mathcal{R}_{g}$ are the ratios of the integrated bottom drag dissipation using the nearbottom velocities accounting for a BBL shear (with the subscript "b") and total veloc- 
ities on top of the BBL $\left(V_{\infty}\right)$. The top of the BBL is chosen as an approximate representation of previous estimates using measured velocities away from the bottom, although this comparison is not exact due to the range of criteria used in prior studies. We provide two estimates of the bottom stress using near-bottom velocities in the dissipation calculation, one using the total velocity $(\mathcal{R})$ and the other one using the geostrophic velocity $\left(\mathcal{R}_{g}\right)$. An implicit assumption underlying the calculation is that the magnitude and spatial variability of bottom drag coefficient $C_{d}$ is well known. This is often not the case and the uncertainty associated with $C_{d}$ can be as large as an order of magnitude. However, this is beyond the scope of our exploration here as we are primarily concerned with the velocity structures near the bottom. Again, the ratios introduced above highlight two important distinctions from previous estimates: i) the reduction of the flow magnitude through the BBL by thermal wind shear; ii) the KE dissipation associated with the geostrophic flow rather than a full velocity, a more relevant and appropriate quantity compared with the wind work on the surface geostrophic motions.

We restrict our analyses over the continental slope region shallower than $3000 \mathrm{~m}-$ where the strong boundary currents reside (Fig. S2) - as this is where Ekman theory predicts the largest effects. We also exclude regions shallower than $200 \mathrm{~m}$ as these regions are excluded from most previous calculations of the wind energy input into the general circulation (e.g. Wunsch, 1998; Scott \& Xu, 2009), and because the effects of atmospheric forcing can reach the BBL, complicating interpretation. The slope region we focus on (between $200 \mathrm{~m}$ and $3000 \mathrm{~m}$ ) has an integrated near-bottom KE approximately equal to the vast abyss below $3000 \mathrm{~m}$, despite the slope region having an area of only $12 \%$ of the abyss, making it disproportionately energetic in the general circulation. Importantly, the point-wise dissipation ratio $V_{b}^{2} \cdot V_{g b} / V_{\infty}^{3}$ (i.e., the ratio of equations (4) to (3) where $V_{\infty}$ is used in equation (3)) is almost ubiquitously below unity above the $3000 \mathrm{~m}$ isobath, whereas there is more variability in deeper areas (Fig. 1d). This spatial pattern is consistent with the theoretical expectations of Ekman arrest dynamics such that larger velocity reduction is found in regions with large horizontal buoyancy gradient (a combination of strong stratification and steep slope) and BBL thickness (see an example section in Fig. 2a) (Brink \& Lentz, 2010; Ruan et al., 2019). This result contrasts with the finding of C. J. Wright et al. (2013), who found a weak downward increase of KE in the lower $100 \mathrm{~m}$. This difference may result from the moored observations preferentially sampling regions deeper than $3000 \mathrm{~m}$ where weak topographic slopes and stratification tend to reduce the efficiency of the Ekman adjustment process (Fig. 1d).

Since we are primarily focused on the dissipation associated with balanced mean flows, we decompose the velocity into geostrophic and ageostrophic components. There is a weak mean vertical shear in the interior $($ hab/H $>1)$ due to the presence of large-scale tilting of isopycnals toward the slope, but the tilting is less pronounced in the interior than within the BBL (figure 2). Averaging all the velocity profiles as a function of heightabove-bottom (hab) normalized to the local BBL depth and local velocity above the BBL, as in Fig. $2 \mathrm{~b}$, the bottom-most velocity $V_{b}$ is around $60 \%$ of $V_{\infty}$. Because of the cubic dependence of the bottom drag dissipation on velocity, this velocity reduction implies $R=0.25$, a $75 \%$ reduction of the total bottom drag dissipation that would otherwise be inferred using $V_{\infty}^{3}$. The reduction of near-bottom velocity through the BBL mostly reflects thermal wind shear due to the horizontal buoyancy gradient, such that $R_{g}=$ 0.22 , implying thermal wind shear in the BBL is significantly reducing the energy lost from the balanced circulation through bottom drag.

\section{Estimates from a high-resolution numerical model of the Atlantic}

The same model is used to provide $\mathcal{R}$ and $\mathcal{R}_{g}$ estimates for the whole Atlantic. To provide a more complete picture, we calculate $\mathcal{R}$ using $V_{g b}$ at the bottom-most grid point as before and $V_{\infty}$ now at four different levels. Specifically, $V_{\infty}$ is estimated using two different definitions of the BBL with density thresholds of 0.01 and $0.02 \mathrm{~kg} \mathrm{~m}^{-3}$, with the 

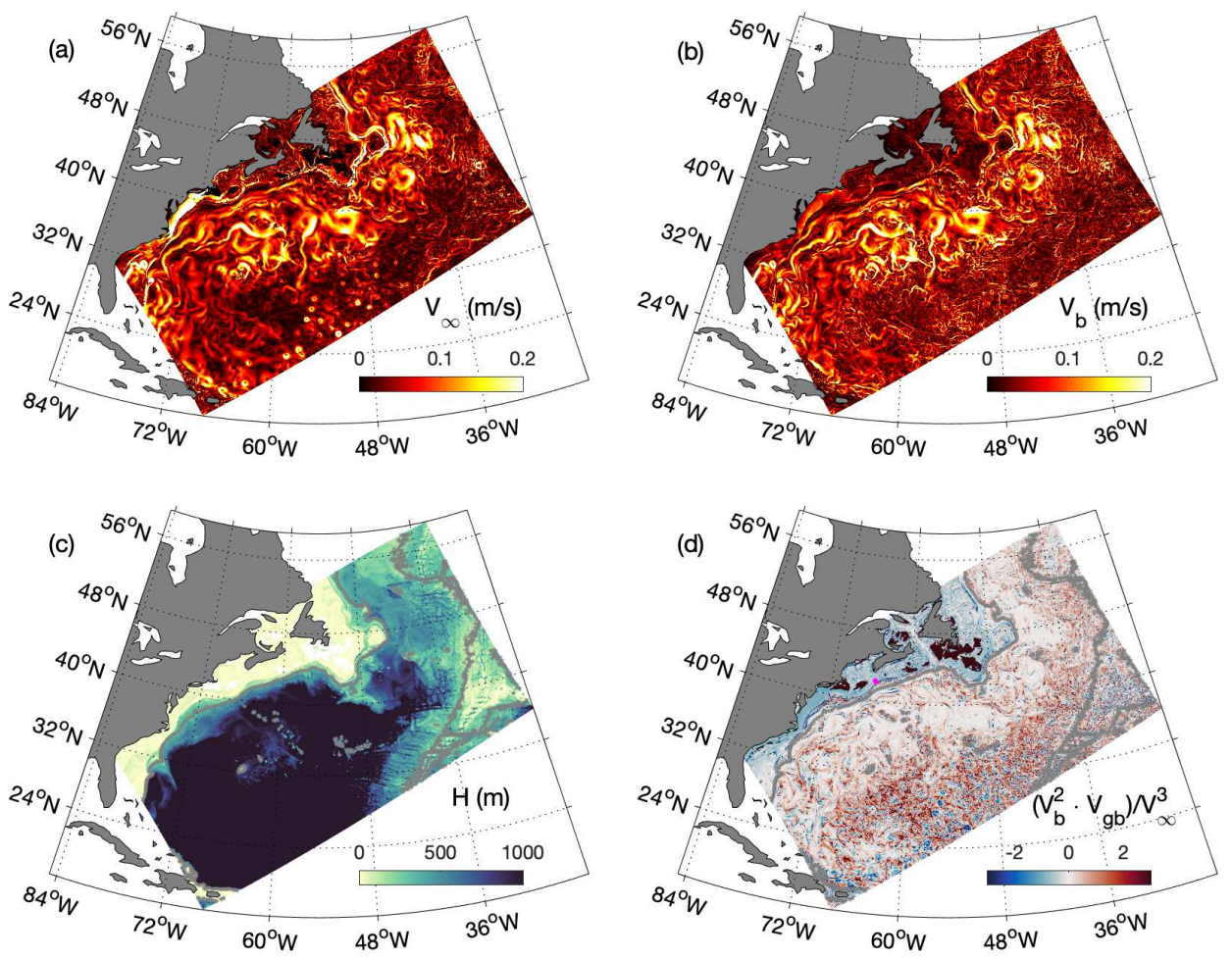

Figure 1. The distribution of velocity at the top of the bottom boundary layer $\left(V_{\infty}\right)(\mathrm{a})$, and at the bottom-most grid point $\left(V_{b}\right)(\mathrm{b})$, the bottom boundary layer thickness $(\mathrm{H})$ (c), and $\log _{10}\left(V_{b}^{2} \cdot V_{g b} / V_{\infty}^{3}\right)$ (d) in the zoomed Atlantic domain. The gray curves in panels (c), (d) denote the $3000 \mathrm{~m}$ isobath. The pink dot (near $42^{\circ} \mathrm{N}, 65^{\circ} \mathrm{W}$ ) in panel (d) (over the continental slope) represents the location of the short transect shown in Fig. 2(a). 

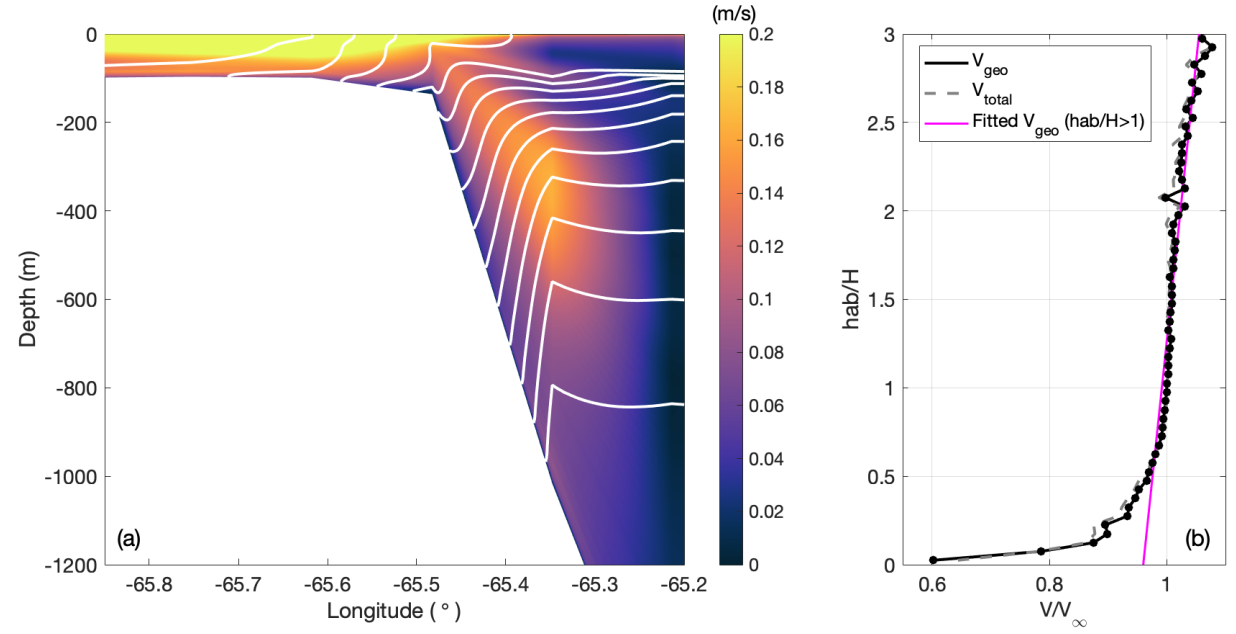

Figure 2. (a) An example transect over the shelf break demonstrating the reduction of flow magnitude associated with isopycnal tilting and thermal wind shear. Color shading represents the magnitude of velocity and white contours denote isopycnals $\left(0.1 \mathrm{~kg} / \mathrm{m}^{3}\right.$ interval). The location of the transect is marked in Fig. 1 (d). (b) The median vertical profile of velocity, normalized by their respective values at the top of the bottom boundary layer (BBL), as a function of height above bottom (hab) over the BBL thickness $(\mathrm{H})$ in the zoomed Atlantic domain. The dashed gray and solid black curves denote the total velocity and its geostrophic component. The magenta curve is a fit to the geostrophic profile above the BBL (hab/H>1) extended to the bottom.

latter a common choice in field studies (e.g. Ruan et al., 2017), and at fixed-depth intervals $(50$ and $100 \mathrm{~m}$ ) away from the bottom to illustrate the influence of instrument spacing, and near-bottom resolution in numerical models, on the dissipation estimate.

The distribution of $\mathcal{R}$ for the whole Atlantic is shown in Fig. 3. To achieve a more reliable calculation, we exclude the equatorial band ( $5^{\circ}$ from the equator) to avoid unrealisticallylarge geostrophic velocities due to the small Coriolis frequency. Similar to before, we also limit our calculations to regions deeper than $200 \mathrm{~m}$ to avoid direct atmospheric forcing and shallower than $3000 \mathrm{~m}$ where the geostrophic currents are strong; inspection of the profiles deeper than $3000 \mathrm{~m}$ indicates that they are often not associated with a true wellmixed BBL, and instead are associated with the very weak abyssal stratification of the quiescent interior (Fig. 3).

Significant reductions can be generally seen along the continental margins, consistent with the results in our zoomed domain (Fig. 3). The ratio in the interior is noisy (Fig. 3) but is excluded from the integrated value of $\mathcal{R}$ since they are deeper than $3000 \mathrm{~m}$. We summarize $\mathcal{R}$ with four different levels for $V_{\infty}$ in Table 1 . The reduction of the dissipation estimates are $56 \%$ and $59 \%$ using $\Delta \rho=0.01 \mathrm{~kg} \mathrm{~m}^{-3}$ and $\Delta \rho=0.02 \mathrm{~kg} \mathrm{~m}^{-3}$ respectively, implying a significant reduction compared to the bottom drag dissipation estimated using the flow on top of the BBL. $\mathcal{R}_{g}$ corresponding to $\Delta \rho=0.01 \mathrm{~kg} \mathrm{~m}^{-3}$ is 0.67 , indicating a non-negligible reduction using geostrophic flows alone. These $\mathcal{R}$ values are associated with 5-day averaged velocities centered at a specific time; we have performed similar analyses using a number of other output in different years and seasons but the results do not vary significantly (Table S1). 

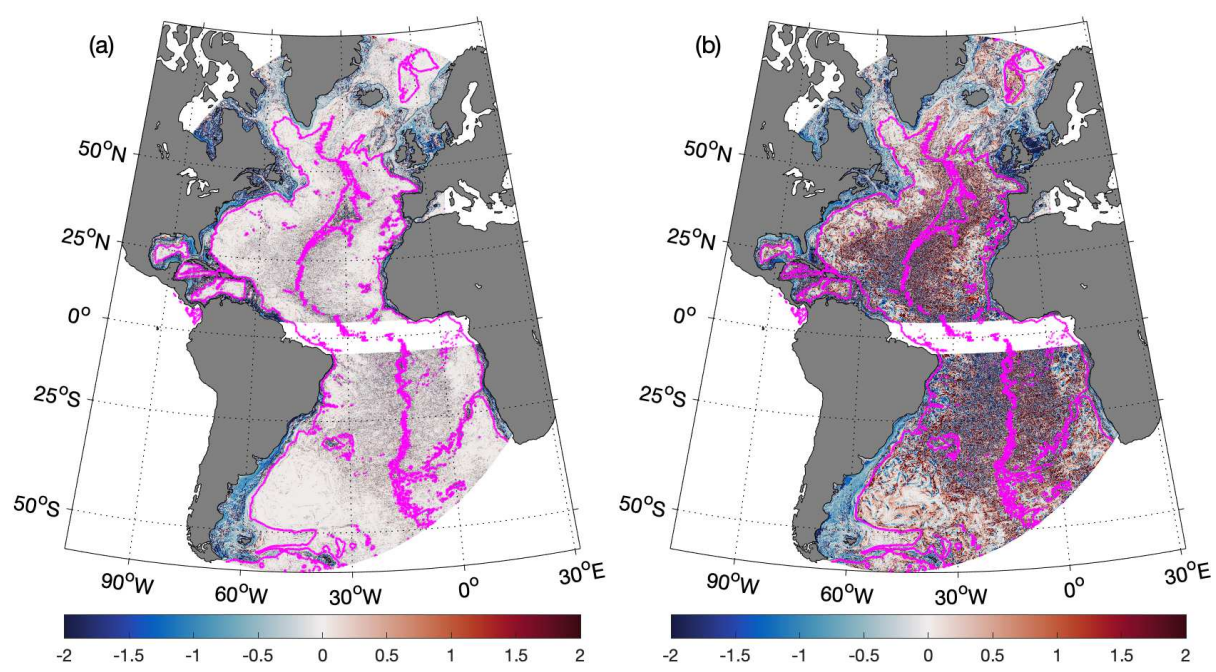

Figure 3. The distribution of $\log _{10}\left(\frac{V_{b}^{2} \cdot V_{g b}}{V_{\infty}^{3}}\right)$ using $V_{\infty}$ at (a) $50 \mathrm{~m}$ away from the bottom (b) the top of the BBL defined using a $\delta \rho=0.01 \mathrm{~kg} \mathrm{~m}^{-3}$ threshold in the whole Atlantic domain. The magenta lines represent the $3000 \mathrm{~m}$ isobath that delineate strong reduction further inshore, which was included in the calculation.

Table 1. Summary of the diagnosed $\mathcal{R}$ using the geostrophic velocity at the bottom and total velocities at four other depths with fixed depth and density interval thresholds.

\begin{tabular}{ccccc} 
& $\Delta z=50 \mathrm{~m}$ & $\Delta z=100 \mathrm{~m}$ & $\Delta \rho=0.01 \mathrm{~kg} \mathrm{~m}^{-3}$ & $\Delta \rho=0.02 \mathrm{~kg} \mathrm{~m}^{-3}$ \\
\hline $\mathcal{R}$ & 0.49 & 0.39 & 0.44 & 0.41 \\
$\mathcal{R}_{\mathrm{g}}$ & 0.74 & 0.59 & 0.67 & 0.63
\end{tabular}




\section{Conclusions}

Using high-resolution numerical models with resolved BBL structures, we demonstrated a significant reduction of the estimated bottom drag dissipation due to vertical shear in the BBL. This reduction results from Ekman processes which generate ageostrophic flows and lead to a state of partial Ekman arrest, where enhanced thermal wind shear in the BBL reduces the near-bottom velocity. Using a density threshold of $\Delta \rho=0.01 \mathrm{~kg} \mathrm{~m}^{-3}$, we showed that the bottom drag dissipation is only 0.44 of what would be calculated using the velocity on top of the BBL. Even using flows only $50 \mathrm{~m}$ away from the bottoma distance much shorter than the near-bottom resolution in typical global ocean models or the spacing between in situ moored instruments - this ratio is still less than 0.5, highlighting the importance of resolving the BBL to accurately estimate the bottom drag dissipation.

These findings suggest that previous global estimates of the bottom drag dissipation are likely biased high by unresolved BBL shear and the robust decrease evident in Table 1 suggests that prior estimates may be biased high by $30-60 \%$. Using bottom moored current meters, C. Wright et al. (2012) estimated 40-56 GW of bottom drag dissipation at the Atlantic zonal boundaries (between $15 \mathrm{~N}$ and $60 \mathrm{~N}$ ). Using the same parameters $C_{d}=0.0025$ and $\rho_{0}=1035 \mathrm{~kg} \mathrm{~m}^{-3}$, our estimates of bottom drag dissipation around the same location are $38.3 \mathrm{TW}$ and $40.9 \mathrm{TW}$ using $V_{\infty}$ at $100 \mathrm{~m}$ above the bottom and at the top of the BBL using $\Delta \rho=0.01 \mathrm{~kg} \mathrm{~m}^{-3}$, respectively. Our estimates fall close to the lower end of C. Wright et al. (2012)'s range and this could be due to i) our choice of $V_{\infty}$, which is closer to the bottom than the current meters; ii) the exclusion of depths between 100-200 m depth in our estimate compared with C. Wright et al. (2012), which could include strong boundary currents that are more directly influenced by atmospheric forcing. Nonetheless, when considering the BBL shear, equation (4) only yields $15.5 \mathrm{GW}$ of bottom drag dissipation within the same area in our model, a reduction of about $60 \%$.

Direct comparison between our estimates and prior work over the global ocean is made difficult by the various criteria that have been applied to select "near-bottom" velocities from observations, varying model vertical resolution, and differences in spatial coverage between observations and models. To the extent that our findings from the Atlantic can be applied to the global integral, our calculation would suggest a revised global estimate of the bottom drag dissipation in the range of $0.04-0.5 \mathrm{TW}$ (found by adjusting the previously estimated range of 0.1-0.7 TW according to the values presented in Table 1). We emphasize however that the effects of Ekman adjustment on bottom drag are most pronounced in regions of strong stratification and steep slopes, hence the direct extrapolation of the Atlantic results to the global ocean should be viewed with skepticism. Of particular interest is determining whether a similar reduction of bottom drag is found along continental margins in the Southern Ocean where the swift Antarctic Circumpolar Current contributes a significant amount of the global KE dissipation. Lastly, the comparison between different simulations is also affected by the detailed numerical implementation, for instance the distance of the bottom-most grid to the bottom topography through which the parameterized drag coefficient can vary (such that in this model the mean value is 0.0037 with standard deviation of 0.0012 ); it can also be sensitive to the parameterizations used in the numerical models, which can influence the general partition of global KE budget and thus the respective dissipation rates (e.g. Trossman et al., 2013, 2016; Pearson et al., 2017).

One potential strategy for addressing the discrepancy between the true near-bottom velocities and those measured at larger height would be through a reduction of the drag coefficient in the bulk formula, which is similar to the strategy employed in existing parameterizations of the drag coefficient across the log-layer (although those are not designed to apply across the entire BBL). It is currently unclear how best to do this for a velocity reduction that is highly variable in both space and time, and instead, development of a physically-motivated parameterization of the near-bottom velocity reduc- 
tion due to the physics of Ekman buoyancy adjustment may be preferable. Indeed, assuming that the bottom velocity decays in time during Ekman adjustment such that it can be written as $V_{b}=\varphi(t) V_{\infty}$, equation (3) implies that the time-averaged bottom drag dissipation should go as $\overline{\mathcal{D}} \approx \overline{\varphi^{3}} \rho_{0} C_{d} V_{\infty}^{3}$ where the overline indicates time averaging over the arrest timescale $T_{e}$ (consistent with the empirical finding of Umlauf et al., 2015). Application of this approach requires knowledge of the functional form of $\varphi$, and the proper timescale for averaging, however it does offer hope for simple corrections to the average bottom drag dissipation calculated using interior velocities. Idealized modeling by Ruan et al. $(2019,2021)$ also suggests the possibility that the true instantaneous bottom stress could be related to modeled or observed interior quantities (such as interior stratification and velocity) through the ratio of the BBL thickness to the theoretical full Ekman arrest thickness. This would allow bottom drag estimates to be corrected for the unresolved effects of partial Ekman arrest in the BBL. Extending these approaches to a full-parameterization requires additional development, but would potentially allow for a more complete assessment of the global integral of the bottom drag dissipation and its effect on ocean circulation.

Despite the considerable remaining quantitative uncertainty in the global estimates, the robustness of the reduction of the BBL dissipation found here for the Atlantic model domain suggests that bottom drag dissipation may play a smaller role than previously hypothesized in removing KE from the balanced flow. Other mechanisms, including submesoscale instabilities of the BBL and the generation and breaking of internal waves near rough bathymetry, may be key to closing this gap. Submesoscale instabilities of the BBL in particular are not resolved even in the high-resolution North Atlantic domain considered here (Wenegrat et al., 2018; Dong et al., 2020). These instabilities may affect the BBL dissipation both indirectly by modifying the dynamics of the Ekman adjustment process, and directly by offsetting a portion of the reduction in bottom drag dissipation through enhanced dissipation of balanced kinetic energy (Wenegrat \& Thomas, 2020). Future work considering both the vertical and horizontal finescale evolution of the BBL will help to constrain the global pathways through which the balanced kinetic energy in the ocean is dissipated through quadratic bottom drag and other mechanisms.

\section{Acknowledgments}

The authors thank Mattias Green for helpful discussion during the preparation of this work, and gratefully acknowledge helpful suggestions from Brian Arbic and an anonymous reviewer. J.O.W. was partially supported by NSF OCE-1756118 and OCE-1948953. J.G. gratefully acknowledges support from the French National Agency for Research (ANR) through the project DEEPER (ANR19CE01000201). J.G. acknowledges PRACE and GENCI for awarding access to HPC resources Joliot-Curie Rome and SKL from GENCITGCC (Grants 2020-A0090112051, 2019gch0401 and PRACE project 2018194735) and HPC facilities DATARMOR of Ple de Calcul Intensif pour la Mer at Ifremer Brest France. The authors thank Gildas Cambon and Sbastien Theetthen for their contribution in the development of the realistic numerical simulation GIGATL3. The information about the simulations can be found at https://doi.org/10.5281/zenodo.4948523. The data used in this study can be obtained at http://doi.org/10.5281/zenodo.4773369.

\section{References}

Arbic, B. K., \& Flierl, G. R. (2004). Baroclinically Unstable Geostrophic Turbulence in the Limits of Strong and Weak Bottom Ekman Friction: Application to Midocean Eddies. Journal of Physical Oceanography, 34, 2257-2273. doi: 10.1175/1520-0485(2004)034〈2257:BUGTIT $\rangle 2.0 . C O ; 2$

Arbic, B. K., \& Scott, R. B. $\quad$ (2008, January). On Quadratic Bottom Drag, Geostrophic Turbulence, and Oceanic Mesoscale Eddies. Journal of Physical Oceanography, 38(1), 84-103. Retrieved 2021-03-25, from http://journals 
.ametsoc.org/doi/10.1175/2007JP03653.1 doi: 10.1175/2007JPO3653.1

Arbic, B. K., Shriver, J. F., Hogan, P. J., Hurlburt, H. E., McClean, J. L., Metzger, E. J., .. Wallcraft, A. J. (2009, February). Estimates of bottom flows and bottom boundary layer dissipation of the oceanic general circulation from global high-resolution models. Journal of Geophysical Research, 114(C2), C02024. Retrieved 2019-07-17, from http://doi.wiley.com/10.1029/ 2008JC005072 doi: 10.1029/2008JC005072

Becker, J., Sandwell, D., Smith, W., Braud, J., Binder, B., Depner, J., . . others (2009). Global bathymetry and elevation data at 30 arc seconds resolution: Srtm30_plus. Marine Geodesy, 32(4), 355-371.

Brink, K. H., \& Lentz, S. J. (2010, April). Buoyancy Arrest and Bottom Ekman Transport. Part I: Steady Flow. Journal of Physical Oceanography, 40(4), 621635. Retrieved 2017-11-10, from http://journals.ametsoc.org/doi/abs/10 .1175/2009JP04266.1 doi: 10.1175/2009JPO4266.1

Canuto, V. M., Howard, A., Cheng, Y., \& Dubovikov, M. (2001). Ocean turbulence. part i: One-point closure modelmomentum and heat vertical diffusivities. Journal of Physical Oceanography, 31(6), 1413-1426.

Carton, J. A., \& Giese, B. S. (2008). A reanalysis of ocean climate using simple ocean data assimilation (soda). Monthly weather review, 136(8), 2999-3017.

Dong, J., Fox-Kemper, B., Zhang, H., \& Dong, C. （2020, September). The Scale of Submesoscale Baroclinic Instability Globally. Journal of Physical Oceanography, 50(9), 2649-2667. Retrieved 2021-04-19, from https:// journals.ametsoc.org/view/journals/phoc/50/9/jpoD200043.xml doi: 10.1175/JPO-D-20-0043.1

Ferrari, R., \& Wunsch, C. (2009, January). Ocean Circulation Kinetic Energy: Reservoirs, Sources, and Sinks. Annual Review of Fluid Mechanics, 41(1), 253-282. Retrieved 2019-07-17, from http://www . annualreviews.org/doi/ 10.1146/annurev.fluid.40.111406.102139 doi: 10.1146/annurev.fluid.40 .111406 .102139

Gula, J., Molemaker, M. J., \& McWilliams, J. C. ～(2016, September). Topographic generation of submesoscale centrifugal instability and energy dissipation. Nature Communications, 7, 12811. Retrieved 2017-05-31, from http://www.nature.com/doifinder/10.1038/ncomms12811 doi: $10.1038 /$ ncomms 12811

Huang, C., \& Xu, Y. (2018, June). Update on the Global Energy Dissipation Rate of Deep-Ocean Low-Frequency Flows by Bottom Boundary Layer. Journal of Physical Oceanography, 48(6), 1243-1255. Retrieved 2021-0324, from https://journals.ametsoc.org/view/journals/phoc/48/6/ jpo-d-16-0287.1.xml doi: 10.1175/JPO-D-16-0287.1

MacCready, P., \& Rhines, P. B. (1991, February). Buoyant inhibition of Ekman transport on a slope and its effect on stratified spin-up. Journal of Fluid Mechanics, 223(-1), 631. Retrieved 2017-06-13, from http:// www.journals.cambridge.org/abstract_S0022112091001581 doi: $10.1017 /$ S0022112091001581

MacCready, P., \& Rhines, P. B. $\quad$ (1993, January). Slippery Bottom Boundary Layers on a Slope. Journal of Physical Oceanography, 23(1), 5-22. Retrieved 2017-06-14, from http://journals.ametsoc.org/doi/abs/ $10.1175 / 1520-0485 \% 281993 \% 29023 \% 3 C 0005 \% 3 A$ SBBLOA $\% 3 E 2.0 . C 0 \% 3 B 2$ doi: 10.1175/1520-0485(1993)023<0005:SBBLOA $>2.0 . \mathrm{CO} ; 2$

Nikurashin, M., \& Ferrari, R. (2011, April). Global energy conversion rate from geostrophic flows into internal lee waves in the deep ocean: INTERNAL LEE WAVES IN THE OCEAN. Geophysical Research Letters, 38(8), n/a-n/a. Retrieved 2019-07-17, from http://doi.wiley.com/10.1029/2011GL046576 doi: 10.1029/2011GL046576

Pearson, B., Fox-Kemper, B., Bachman, S., \& Bryan, F. (2017). Evaluation of scale- 
aware subgrid mesoscale eddy models in a global eddy-rich model. Ocean Modelling, 115, 42-58.

Rocha, C. B., Wagner, G. L., \& Young, W. R. (2018). Stimulated generation: extraction of energy from balanced flow by near-inertial waves. Journal of Fluid Mechanics, 847, 417-451.

Ruan, X. (2021). Note on the bulk estimate of the energy dissipation rate in the bottom boundary layer. Retrieved from https://eartharxiv.org/ repository/view/2297/ doi: 10.31223/X5090H

Ruan, X., Thompson, A. F., Flexas, M. M., \& Sprintall, J. (2017, October). Contribution of topographically generated submesoscale turbulence to Southern Ocean overturning. $\quad$ Nature Geoscience, 10(11), 840-845. Retrieved 201712-03, from http://www.nature.com/doifinder/10.1038/ngeo3053 doi: $10.1038 /$ ngeo3053

Ruan, X., Thompson, A. F., \& Taylor, J. R. (2019, February). The Evolution and Arrest of a Turbulent Stratified Oceanic Bottom Boundary Layer over a Slope: Downslope Regime. Journal of Physical Oceanography, 49(2), 469-487. Retrieved 2019-07-17, from http://journals.ametsoc.org/doi/10.1175/ JPO-D-18-0079.1 doi: 10.1175/JPO-D-18-0079.1

Ruan, X., Thompson, A. F., \& Taylor, J. R. (2021, April). The Evolution and Arrest of a Turbulent Stratified Oceanic Bottom Boundary Layer over a Slope: Upslope Regime and PV Dynamics. Journal of Physical Oceanography, 51(4), 1077-1089. Retrieved from https://journals.ametsoc.org/view/journals/ phoc/51/4/JPO-D-20-0168.1.xml doi: 10.1175/JPO-D-20-0168.1

Saha, S., Moorthi, S., Pan, H.-L., Wu, X., Wang, J., Nadiga, S., . . others （2010). The ncep climate forecast system reanalysis. Bulletin of the American Meteorological Society, 91 (8), 1015-1058.

Scott, R. B., Goff, J. A., Naveira Garabato, A. C., \& Nurser, A. J. G. September). Global rate and spectral characteristics of internal gravity wave generation by geostrophic flow over topography. Journal of Geophysical Research, 116(C9), C09029. Retrieved 2019-07-17, from http://doi.wiley.com/ 10.1029/2011JC007005 doi: 10.1029/2011JC007005

Scott, R. B., \& Xu, Y. (2009, March). An update on the wind power input to the surface geostrophic flow of the World Ocean. Deep Sea Research Part I: Oceanographic Research Papers, 56 (3), 295-304. Retrieved 2019-07-17, from https://linkinghub.elsevier.com/retrieve/pii/S0967063708001994 doi: $10.1016 /$ j.dsr.2008.09.010

Sen, A., Scott, R. B., \& Arbic, B. K. (2008, May). Global energy dissipation rate of deep-ocean low-frequency flows by quadratic bottom boundary layer drag: Computations from current-meter data. Geophysical Research Letters, 35(9), L09606. Retrieved 2019-07-28, from http://doi.wiley.com/10.1029/ 2008GL033407 doi: 10.1029/2008GL033407

Shchepetkin, A. F., \& McWilliams, J. C. (2005, January). The regional oceanic modeling system (ROMS): a split-explicit, free-surface, topography-followingcoordinate oceanic model. Ocean Modelling, 9(4), 347-404. Retrieved 2017-04-11, from http://linkinghub.elsevier.com/retrieve/pii/ S1463500304000484 doi: 10.1016/j.ocemod.2004.08.002

Taylor, G. I. (1920). I. tidal friction in the irish sea. Philosophical Transactions of the Royal Society of London. Series A, Containing Papers of a Mathematical or Physical Character, 220(571-581), 1-33.

Thompson, A. F., \& Young, W. R. (2006, April). Scaling Baroclinic Eddy Fluxes: Vortices and Energy Balance. Journal of Physical Oceanography, 36(4), 720-738. Retrieved 2021-03-25, from http://journals.ametsoc.org/doi/ 10.1175/JP02874.1 doi: 10.1175/JPO2874.1

Trossman, D. S., Arbic, B. K., Garner, S. T., Goff, J. A., Jayne, S. R., Metzger, E. J., \& Wallcraft, A. J. (2013). Impact of parameterized lee wave drag on 
the energy budget of an eddying global ocean model.

Ocean Modelling, 72, $119-142$.

Trossman, D. S., Arbic, B. K., Richman, J. G., Garner, S. T., Jayne, S. R., \& Wallcraft, A. J. (2016). Impact of topographic internal lee wave drag on an eddying global ocean model. Ocean Modelling, 97, 109-128.

Trossman, D. S., Arbic, B. K., Straub, D. N., Richman, J. G., Chassignet, E. P., Wallcraft, A. J., \& Xu, X. (2017, August). The Role of Rough Topography in Mediating Impacts of Bottom Drag in Eddying Ocean Circulation Models. Journal of Physical Oceanography, 47(8), 1941-1959. Retrieved 202103-25, from https://journals.ametsoc.org/view/journals/phoc/47/8/ jpo-d-16-0229.1.xml doi: 10.1175/JPO-D-16-0229.1

Umlauf, L., \& Burchard, H. (2003). A generic length-scale equation for geophysical turbulence models. Journal of Marine Research, 61 (2), 235-265.

Umlauf, L., Smyth, W. D., \& Moum, J. N. (2015, December). Energetics of Bottom Ekman Layers during Buoyancy Arrest. Journal of Physical Oceanography, 45(12), 3099-3117. Retrieved 2017-06-21, from http://journals.ametsoc .org/doi/10.1175/JPO-D-15-0041.1 doi: 10.1175/JPO-D-15-0041.1

Wenegrat, J. O., Callies, J., \& Thomas, L. N. （2018, November). Submesoscale Baroclinic Instability in the Bottom Boundary Layer. Journal of Physical Oceanography, 48(11), 2571-2592. Retrieved 2019-05-21, from http://journals.ametsoc.org/doi/10.1175/JPO-D-17-0264.1 doi: 10.1175/JPO-D-17-0264.1

Wenegrat, J. O., \& Thomas, L. N. ～(2020, June). Centrifugal and Symmetric Instability during Ekman Adjustment of the Bottom Boundary Layer. Journal of Physical Oceanography, 50(6), 1793-1812. Retrieved 202006-22, from https://journals.ametsoc.org/jpo/article/50/6/1793/ 346599/Centrifugal-and-Symmetric-Instability-during-Ekman doi: 10.1175/JPO-D-20-0027.1

Wright, C., Scott, R., Arbic, B. K., \& Furnival, D. (2012). Bottom dissipation of subinertial currents at the atlantic zonal boundaries. Journal of Geophysical Research: Oceans, $117(\mathrm{C} 3)$.

Wright, C. J., Scott, R. B., Ailliot, P., \& Furnival, D. (2014, April). Lee wave generation rates in the deep ocean. Geophysical Research Letters, 41(7), 2434-2440. Retrieved 2019-07-17, from http://doi.wiley.com/10.1002/2013GL059087 doi: 10.1002/2013GL059087

Wright, C. J., Scott, R. B., Furnival, D., Ailliot, P., \& Vermet, F. (2013, February). Global Observations of Ocean-Bottom Subinertial Current Dissipation. Journal of Physical Oceanography, 43(2), 402-417. Retrieved 2019-07-22, from http://journals.ametsoc.org/doi/abs/10.1175/JPO-D-12-082.1 doi: 10.1175/JPO-D-12-082.1

Wunsch, C. (1998). The Work Done by the Wind on the Oceanic General Circulation. Journal of Physical Oceanography, 28(11), 2332-2340. Retrieved from https : //doi .org/10.1175/1520-0485(1998) 028<2332: TWDBTW>2.0.C0;2 doi: 10.1175/1520-0485(1998)028<2332:TWDBTW $\rangle 2.0 . C O ; 2$ 